\title{
Cunninghamella blakesleeana-mediated biotransformation of a contraceptive drug, desogestrel, and anti-MDR-Staphylococcus aureus activity of its metabolites
}

\author{
Atia-tul-Wahab $^{\mathrm{a}, *}$, Mahwish Siddiqui ${ }^{\mathrm{b}}$, Iman Ibrahim $^{\mathrm{c}, \mathrm{d}}$, Arifa Hussain ${ }^{\mathrm{b}}$, El Hassan Ajandouz ${ }^{\mathrm{c}}$, \\ Akram Hijazi $^{\mathrm{d}}$, Elias Baydoun ${ }^{\mathrm{e}, *}$, M. Iqbal Choudhary ${ }^{\mathrm{a}, \mathrm{b}, \mathrm{f}}$ \\ ${ }^{a}$ Dr. Panjwani Center for Molecular Medicine and Drug Research, International Center for Chemical and Biological Sciences, University of Karachi, Karachi 75270, Pakistan \\ ${ }^{\mathrm{b}}$ H. E. J. Research Institute of Chemistry, International Center for Chemical and Biological Sciences, University of Karachi, Karachi 75270, Pakistan \\ c Aix Marseille Universite, CNRS, Centrale Marseille, iSm2, Marseille, France \\ ${ }^{\mathrm{d}}$ Doctoral School of Science and Technology, Research Platform for Environmental Science (PRASE), Lebanese University, Lebanon \\ e Department of Biology, American University of Beirut, Beirut 1107 2020, Lebanon \\ ${ }^{\mathrm{f}}$ Department of Biochemistry, Faculty of Science, King Abdulaziz University, Jeddah 21412, Saudi Arabia
}

\section{A R T I C L E I N F O}

\section{Article history:}

Received 20 October 2017

Revised 27 December 2017

Accepted 29 December 2017

Available online 2 January 2018

\section{Keywords:}

Drug resistant Staphylococcus aureus

Desogestrel

Biotransformation

Cunninghamella blakesleeana

$6 \beta, 15 \beta$-Dihydroxy-desogestrel

Anti-bacterial

Cytotoxicity

\begin{abstract}
A B S T R A C T
Staphylococcus aureus is one of the most infectious agents among staphylococcal bacteria. Currently many strains of $S$. aureus have developed resistance against available antibiotics. Therefore, the treatment of infections caused by them is a major challenge. During current study, desogestrel (1), a contraceptive drug, was found to be a potent growth inhibitor of drug resistant strains of S. aureus. Therefore, in search of new and effective agents against multi-drug resistant $S$. aureus strains, whole-cell bio-catalytic conversion of desogestrel (1) by Cunninghamella blakesleeana ATCC $8688 \mathrm{~A}$ at pH 7.0 and $25^{\circ} \mathrm{C}$ was carried out, yielding three new metabolites, 13 -ethyl-11-methylene-18,19-dinor-17 $\alpha$-pregn-4-en-20-yn-6 $\beta, 15 \beta, 17 \beta$ triol (2), 13-ethyl-11-methylene-18,19-dinor-17 $\alpha$-pregn-4-en-20-yn-3 $\beta, 6 \beta, 17 \beta$-triol (3), and 13-ethyl-1 1-methylene-18,19-dinor-17 $\alpha$-pregn-20-yn-3 $\alpha, 5 \alpha, 6 \beta, 17 \beta$-tetraol (4), along with a known metabolite, 1 3-ethyl-11-methylene-18,19-dinor-17 $\alpha$-pregn-4-en-20-yn-6 $\beta, 17 \beta$-dihydroxy-3-one (5). Among them, compounds 1-2 showed a potent activity against S. aureus EMRSA-17, S. aureus NCTC 13277 (MRSA252), and S. aureus NCTC 13143, and clinically isolated Pakistani strain of S. aureus in an in vitro Microplate Alamar Blue Assay (MABA). Vancomycin was used as the standard drug in this assay. In addition, compound $\mathbf{1}$ also showed a significant activity against vancomycin-resistant $S$. aureus (VRSA) ATCC 700699. Compounds 1-5 were also evaluated against 3T3 normal cell line (mouse fibroblast) where they all were identified as non-cytotoxic. The present study thus provides new leads for the development of anti-bacterial drugs against MDR S. aureus.
\end{abstract}

(c) 2018 Elsevier Inc. All rights reserved.

\section{Introduction}

Due to the emergence of resistance against available antibiotics, and rapid pathogenic mutation rate, the treatment of bacterial infections have become a global health challenge [1,2]. S. aureus, gram positive bacteria, is an etiological agent of many infections, ranging from mild to life threatening infections, e.g., skin and soft-tissue infections, blood stream infections, pneumonia, etc. It is the most common causative agent of blood stream infections in the Canada, United States, and Latin America. Each year, around

\footnotetext{
* Corresponding authors.

E-mail addresses: atia.tulwahab@iccs.edu ( Atia-tul-Wahab), eliasbay@aub.edu. lb (E. Baydoun).
}

50,000 patients die in the USA alone due to $S$. aureus infections [3-7]. Asia is also a region with the highest prevalence of drug resistant S. aureus infections worldwide. The dissemination of anti-biotic resistant $S$. aureus infections are serious public health problems in many countries of Asia [8].

Natural products, such as steroids, terpenes, and flavonoids have been the most effective compounds for the treatment of many diseases, including cancer, immune system disorders, cardiovascular diseases, and bacterial, viral and fungal infections since several decades. Due to the presence of many asymmetric centers and inert hydrocarbon skeleton, derivatization of natural products is often a challenging task through conventional methodologies. However, biotransformation by microorganisms, i.e., bacteria or fungi, and plant cell cultures and pure enzymes is an effective and efficient 
approach for the synthesis of structural analogues of natural products. Resulting products are regio-, stereo-, and chemo-selective due to the catalytic action of a range of enzymes. At present, biotransformation techniques are successfully used in various fields, such as medicines, agriculture, polymer, and food industry, etc. due to selective nature of biological catalysts (enzymes) [9-13].

Desogestrel (13-ethyl-11-methylene-18,19-dinor-17 $\alpha$-pregn-4en-20-yn-17-ol) (1) is an orally active third generation contraceptive steroidal drug. It is a synthetic progestin, used successfully in hormonal contraceptives with low or no androgenic activity. The rational to develop this synthetic progestin was to replace the use of classical oral progestogens that possess androgenic effects, specifically harmful effects on plasma lipids. Like traditional progestogenonly pills (POPs) containing $30 \mu \mathrm{g}$ levonorgestrel, and $75 \mu \mathrm{g}$ desogestrel also have low effects on the metabolism of lipids. Therefore, the POPs have been marketed for over three decades. However, due to lower contraceptive efficacy, and random bleeding pattern, POPs have been less accepted than the combined oral contraceptive pills (COCPs) by the prescribers, and consumers [14-16].

In continuation of our work on fungal-mediated synthesis of bioactive steroids [17-20], including $17 \alpha$-ethynyl substituted steroidal drugs [21-23], an oral contraceptive drug, desogestrel
(1) $\left(\mathrm{C}_{22} \mathrm{H}_{30} \mathrm{O}\right)$, was incubated with $\mathrm{C}$. blakesleeana, yielding metabolites 2-5 (Fig. 1). Interestingly, substrate $\mathbf{1}$ and its metabolite $\mathbf{2}$ showed a potent anti-bacterial activity against resistant strains of S. aureus, while metabolites 3-5 showed no growth inhibition of these bacterial strains.

\section{Materials and methods}

\subsection{Fungal cell culture and media}

The fungal cell culture of $C$. blakesleeana (ATCC 8688A) was used for biotransformational study. C. blakesleeana was grown on SDA slants (Sabouraud Dextrose Agar), and maintained at $4{ }^{\circ} \mathrm{C}$.

The media ( $1 \mathrm{~L}$ ) was prepared by adding $10 \mathrm{~g}$ glucose, $5 \mathrm{~g}$ peptone, $5 \mathrm{~g} \mathrm{NaCl}, 5 \mathrm{~g} \mathrm{KH} \mathrm{PO}_{4}$, and $10.0 \mathrm{~mL}$ glycerol in $1 \mathrm{~L}$ distilled water for the maximum growth of $C$. blakesleeana.

\subsection{Bacterial strains and media}

Drug resistant $S$. aureus strains, S. aureus EMRSA-17, S. aureus NCTC 13277, S. aureus NCTC 13143, clinically isolated S. aureus, and vancomycin-resistant S. aureus ATCC 70-069 were targeted

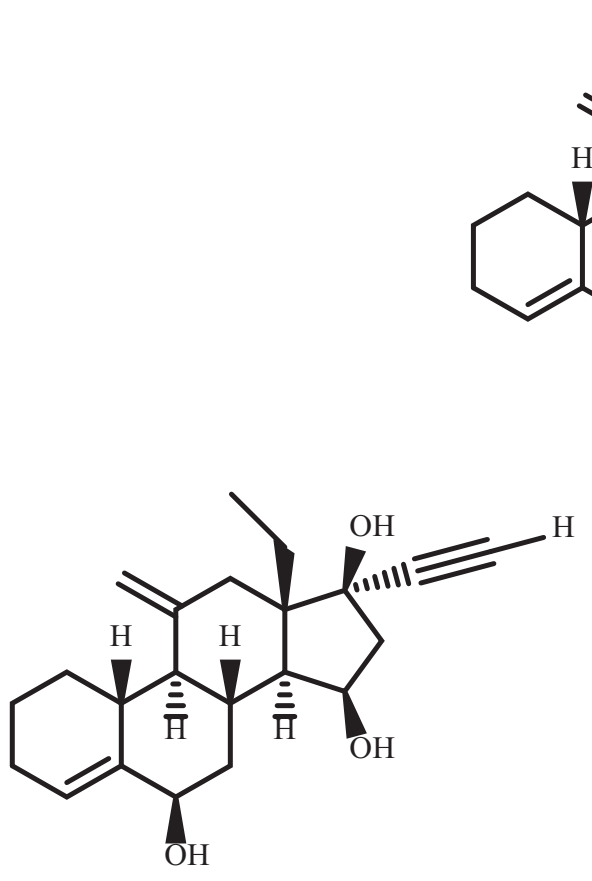

2

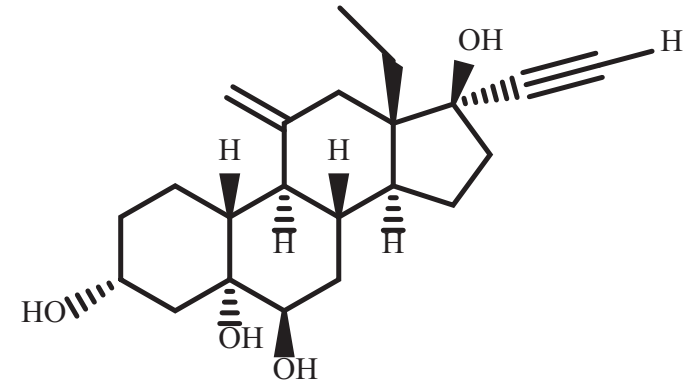

4

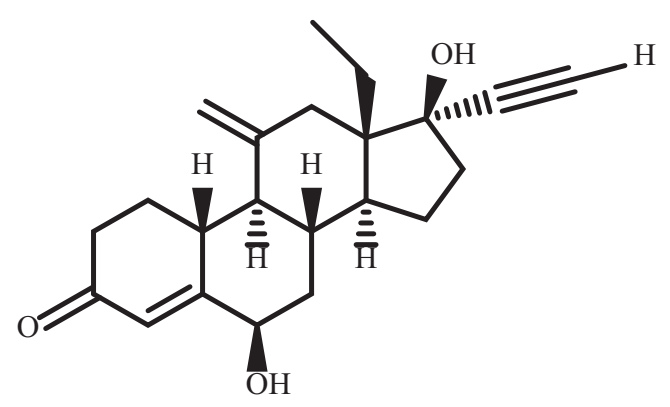

5

Fig. 1. Biotransformation of desogestrel (1) with Cunninghamella blakesleeana. 
Table 1

${ }^{1} \mathrm{H}-\mathrm{NMR}$ chemical shift data $\left(J\right.$ and $W_{1 / 2}$ in $\mathrm{Hz}$ ) of compounds 1-5 ( $\left.\delta \mathrm{ppm}\right)$.

\begin{tabular}{|c|c|c|c|c|c|}
\hline Position & $\mathbf{1}^{\mathrm{a}}$ & $2^{\mathrm{b}}$ & $3^{b}$ & $4^{\mathrm{a}}$ & $5^{\mathrm{a}}$ \\
\hline 1 & $\begin{array}{l}2.23, \mathrm{~m} \\
1.13, \mathrm{~m}\end{array}$ & $\begin{array}{l}\text { 1.63, overlap; } \\
1.55, \mathrm{~m}\end{array}$ & $\begin{array}{l}2.39, \text { overlap; } \\
1.05 \text {, overlap }\end{array}$ & $\begin{array}{l}\text { 1.23, overlap, } \\
2(\mathrm{H})\end{array}$ & $\begin{array}{l}1.94, \mathrm{~m} \\
1.28, \mathrm{~m}\end{array}$ \\
\hline 2 & 1.62, overlap $2[\mathrm{H}]$ & $\begin{array}{l}2.31 \text {, overlap; } \\
1.13 \text {, overlap }\end{array}$ & $\begin{array}{l}1.91 \text {, overlap; } \\
1.38 \text {, overlap }\end{array}$ & $\begin{array}{l}1.91 \text {, overlap; } \\
1.27 \text {, overlap }\end{array}$ & $\begin{array}{l}2.54, \mathrm{~m} \\
1.59, \mathrm{~m}\end{array}$ \\
\hline 3 & 1.92, overlap $2[\mathrm{H}]$ & $\begin{array}{l}\text { 1.91, overlap } \\
2[\mathrm{H}]\end{array}$ & $\begin{array}{l}4.10, \text { overlap } \\
\left(W_{1 / 2}=18.9\right)\end{array}$ & $\begin{array}{l}3.91, \text { br. d } \\
\left(J_{e / e}=2.5\right. \\
\left.W_{1 / 2}=9.6\right)\end{array}$ & - \\
\hline 4 & 5.45 , br. s & 5.71 , br. s & 5.51 , br. s & $\begin{array}{l}1.90 \text {, overlap; } \\
1.64 \text {, overlap }\end{array}$ & 5.92, br. s \\
\hline 5 & - & - & - & - & - \\
\hline 6 & $\begin{array}{l}2.18, \mathrm{~m} ; \\
1.94, \text { overlap }\end{array}$ & $\begin{array}{l}4.18, \text { overlap } \\
\left(W_{1 / 2}=6.4\right)\end{array}$ & $\begin{array}{l}4.15, \text { br. s } \\
\left(W_{1 / 2}=6.3\right)\end{array}$ & $\begin{array}{l}3.41, \text { br. t } \\
\left(J_{6 e / 7 e}=2.5, W_{1 / 2}=6.7\right)\end{array}$ & $\begin{array}{l}4.31, \text { br. t } \\
\left(J_{6 e / 7 e}=2.8, W_{1 / 2}=8.1\right)\end{array}$ \\
\hline 7 & $\begin{array}{l}1.68, \mathrm{~m} \\
0.88, \mathrm{~m}\end{array}$ & $\begin{array}{l}2.13 \text {, overlap; } \\
1.21 \text {, overlap }\end{array}$ & $\begin{array}{l}1.89 \text {, overlap; } \\
1.12 \text {, overlap }\end{array}$ & $\begin{array}{l}1.92 \text {, overlap; } \\
1.52 \text {, overlap }\end{array}$ & $\begin{array}{l}1.94, \mathrm{~m} \\
1.28, \mathrm{~m}\end{array}$ \\
\hline 8 & $2.25, \mathrm{~m}$ & 2.14 , overlap & 2.67 , overlap & 1.89 , overlap & $2.04, \mathrm{~m}$ \\
\hline 9 & $1.81, \mathrm{~m}$ & $1.34, \mathrm{~m}$ & 1.25 , overlap & 1.25 , overlap & 1.52 , overlap \\
\hline 10 & $1.29, \mathrm{~m}$ & 2.66 , overlap & $1.85, \mathrm{~m}$ & 1.88 , overlap & $3.03, \mathrm{~m}$ \\
\hline 11 & - & - & - & - & - \\
\hline 12 & $\begin{array}{l}2.64, \mathrm{~m} \\
2.26, \mathrm{~m}\end{array}$ & $\begin{array}{l}2.68, \text { overlap; } \\
2.22 \text {, overlap }\end{array}$ & $\begin{array}{l}2.65, \text { overlap } \\
2.26, \mathrm{~m}\end{array}$ & $\begin{array}{l}2.64, \text { overlap; } \\
2.27 \text {, overlap }\end{array}$ & $\begin{array}{l}\text { 2.71, overlap; } \\
2.32 \text {, overlap }\end{array}$ \\
\hline 13 & - & - & - & - & - \\
\hline 14 & $1.28, \mathrm{~m}$ & 1.66, overlap & 1.79, overlap & 2.61 , overlap & 1.87, m \\
\hline 15 & $\begin{array}{l}1.63, \mathrm{~m} \\
1.39, \mathrm{~m}\end{array}$ & $\begin{array}{l}4.16, \text { overlap } \\
\left(W_{1 / 2}=5.1\right)\end{array}$ & $\begin{array}{l}1.51, \text { overlap; } \\
1.33, \text { overlap }\end{array}$ & $\begin{array}{l}1.71 \text {, overlap; } \\
1.34 \text {, overlap }\end{array}$ & $\begin{array}{l}1.63, \mathrm{~m} \\
1.37, \mathrm{~m}\end{array}$ \\
\hline 16 & $\begin{array}{l}2.26, \mathrm{~m} \\
2.05, \mathrm{~m}\end{array}$ & $\begin{array}{l}2.71 \text {, overlap; } \\
2.12 \text {, overlap }\end{array}$ & $\begin{array}{l}2.26 \text {, overlap; } \\
2.06 \text {, overlap }\end{array}$ & $\begin{array}{l}2.28, \text { overlap; } \\
2.06 \text {, overlap }\end{array}$ & $\begin{array}{l}2.31 \text {, overlap; } \\
2.10 \text {, overlap }\end{array}$ \\
\hline 17 & - & - & - & - & - \\
\hline 18 & $\begin{array}{l}1.41, \mathrm{~m} \\
1.01, \mathrm{~m}\end{array}$ & $\begin{array}{l}1.55 \text {, overlap; } \\
1.49 \text {, overlap }\end{array}$ & $\begin{array}{l}1.49, \mathrm{~m} \\
1.00, \mathrm{~m}\end{array}$ & $\begin{array}{l}1.62, \text { overlap; } \\
1.38, \text { overlap }\end{array}$ & $1.48, \mathrm{~m} \mathrm{2}[\mathrm{H}]$ \\
\hline 19 & $\begin{array}{l}1.02, \mathrm{t} \\
\left(J_{19 / 18}=7.8\right)\end{array}$ & $\begin{array}{l}1.03, \mathrm{t} \\
\left(J_{19 / 18}=7.8\right)\end{array}$ & $\begin{array}{l}1.01, \mathrm{t} \\
\left(J_{19 / 18}=7.5\right)\end{array}$ & $\begin{array}{l}1.02, \mathrm{t} \\
\left(J_{19 / 18}=7.5\right)\end{array}$ & $\begin{array}{l}1.05, \mathrm{t} \\
\left(J_{19 / 18}=7.5\right)\end{array}$ \\
\hline 20 & $4.95, \mathrm{~s} ; 4.75, \mathrm{~s}$ & $5.01, \mathrm{~s} ; 4.81, \mathrm{~s}$ & $4.99, \mathrm{~s} ; 4.81, \mathrm{~s}$ & $4.98, \mathrm{~s} ; 4.76, \mathrm{~s}$ & $5.05, \mathrm{~s} ; 4.90, \mathrm{~s}$ \\
\hline 21 & - & - & - & - & - \\
\hline 22 & $2.90, \mathrm{~s}$ & $2.91, \mathrm{~s}$ & $2.91, \mathrm{~s}$ & $2.92, \mathrm{~s}$ & $2.91, \mathrm{~s}$ \\
\hline
\end{tabular}

a $500 \mathrm{MHz}$.

b $600 \mathrm{MHz}$.

in this study. All the bacterial strains were grown on SDA slants, and maintained at $4{ }^{\circ} \mathrm{C}$.

\subsection{General experimental conditions}

Desogestrel (1) $\left(\mathrm{C}_{22} \mathrm{H}_{30} \mathrm{O}\right)$ was acquired from Haihang Industry Co., Ltd. (China). Ingredients for media preparation were purchased from Sigma-Aldrich (Germany), Oxoid Ltd. (England), and Daejung Chemicals and Metals Co., Ltd. (Korea). The degree of transformations and purity of compounds were analyzed by using silica coated thin layer chromatographic plates $\left(\mathrm{PF}_{254}\right.$, Merck KGaA, Germany). Fractionations of gummy crude extract was carried out by column chromatography (CC) (Silica gel, 70-230 mesh, E. Merck, Germany). All the fractions were finally purified through RPHPLC (LC-908, Japan), equipped with YMC M-80 (20-250 mm i.d. 4-5 $\mu \mathrm{m}$ ). JASCO P-2000 polarimeter (Japan), Bruker Vector 22 spectrophotometer (France), and Buchi 560 device (Switzerland) were used for the measurements of optical rotations, IR absorbances, and melting points of all purified compounds, respectively. ${ }^{1} \mathrm{H}-(500$, and $600 \mathrm{MHz}),{ }^{13} \mathrm{C}-(125 \mathrm{MHz})$ and 2D-NMR spectra were recorded in $\mathrm{CD}_{3} \mathrm{OD}$ on Bruker Avance-NMR (Switzerland). EI- and HREI-MS of all compounds were determined on Jeol JMS-600H mass spectrometer (Japan), while Evolution 300 UV-visible spectrophotometer (England) was used for recording of UV absorbances. Analytical grade solvents were used in this research work.

\subsection{Fermentation and extraction protocol of desogestrel (1) with $C$. blakesleeana}

Based on small scale screening results, $4 \mathrm{~L}$ media for the growth of $C$. blakesleeana was prepared by mixing aforementioned media ingredients in $4 \mathrm{~L}$ distilled water, and distributed into $250 \mathrm{~mL}$ of 40 Erlenmeyer flasks ( $100 \mathrm{~mL}$ in each), cotton plugged, and autoclaved at $121{ }^{\circ} \mathrm{C}$. This sterilized media was cooled at room temperature, and inoculated with seed flasks of $C$. blakesleeana under sterilized conditions. The flasks were kept for 3-4 days on a shaker (121 rpm) at $25^{\circ} \mathrm{C}$ for growth of fungi. After mature growth of $C$. blakesleeana in each flask, $1 \mathrm{~g}$ of desogestrel $(\mathbf{1})\left(\mathrm{C}_{22} \mathrm{H}_{30} \mathrm{O}\right)$ was dissolved in $20 \mathrm{~mL}$ of methanol, dispensed $(0.5 \mathrm{~mL})$ in each flask, and again placed on a shaker ( $121 \mathrm{rpm})$ at $25{ }^{\circ} \mathrm{C}$ for fourteen days.

After fourteen days incubation period, the reaction was stopped by adding dichloromethane (DCM) in each flasks, and filtered in order to separate fungal mass. The filtrate (organic + aqueous phase) was separated by extracting with 12 L DCM. The organic phase was made moisture free by adding anhydrous sodium sulfate $\left(\mathrm{Na}_{2} \mathrm{SO}_{4}\right)$, filtered, and then concentrated under reduced pressure. This yielded $1.5 \mathrm{~g}$ brownish gummy crude which was fractionated by column chromatography (CC) with a mobile phase of hexanes-acetone. By increasing 5-100\% gradients of acetone, the polarity of mobile phase was increased. As a result, four main fractions (1-4) were obtained which were analyzed by thin layer chromatography (TLCs). The fractions were purified through recycling RP-HPLC. Compounds 2 (methanol-water; 7/3, $R_{T}=29 \mathrm{~min}$ ), 3 (methanol-water; 6/4, $R_{T}=26 \mathrm{~min}$ ), 4 (methanol-water; 6/4, $R_{T}=$ $22 \mathrm{~min}$ ), and 5 (methanol-water; $7 / 3, R_{T}=27 \mathrm{~min}$ ) were purified from fractions $1-4$, respectively, through recycling RP-HPLC.

\subsubsection{3-Ethyl-11-methylene-18,19-dinor-17 $\alpha$-pregn-4-en-20-yn-} $3 \beta-15 \beta-17 \beta$-triol (2)

White solid; m. p. $246-248{ }^{\circ} \mathrm{C} ;[\alpha]_{D}^{25}=-310$ (c 0.001, MeOH); IR $\left(\mathrm{CH}_{3} \mathrm{Cl}\right): v_{\max }\left(\mathrm{cm}^{-1}\right)$ 3393, $3303(\mathrm{OH}), 2926(\mathrm{CH}), 1644(\mathrm{C}=\mathrm{C})$; HREI-MS $m / z$ 342.2179 [M+ $\left(\mathrm{C}_{22} \mathrm{H}_{30} \mathrm{O}_{3}\right.$, calcd. 342.2195); EI-MS 
Table 2

${ }^{13} \mathrm{C}$-NMR chemical shift data of compounds 1-5 ( $\delta$ ppm) at $125 \mathrm{MHz} \mathrm{NMR}$ spectrometer.

\begin{tabular}{llllll}
\hline Carbons & $\mathbf{1}$ & $\mathbf{2}$ & $\mathbf{3}$ & $\mathbf{4}$ & $\mathbf{5}$ \\
\hline 1 & 30.5 & 30.1 & 27.9 & 29.5 & 37.5 \\
2 & 22.8 & 22.7 & 32.3 & 25.2 & 29.0 \\
3 & 26.7 & 26.5 & 67.8 & 69.6 & 203.3 \\
4 & 122.2 & 126.5 & 129.7 & 40.3 & 126.7 \\
5 & 149.3 & 148.4 & 144.0 & 76.1 & 168.4 \\
6 & 36.6 & 74.2 & 73.3 & 74.4 & 72.2 \\
7 & 33.2 & 38.1 & 38.8 & 29.0 & 38.2 \\
8 & 37.9 & 33.6 & 33.5 & 38.7 & 35.9 \\
9 & 53.7 & 56.0 & 56.1 & 47.5 & 54.9 \\
10 & 44.2 & 33.1 & 36.4 & 43.7 & 34.8 \\
11 & 141.0 & 142.1 & 148.5 & 149.5 & 148.2 \\
12 & 41.8 & 41.8 & 41.7 & 41.6 & 41.6 \\
13 & 51.6 & 51.5 & 49.8 & 51.4 & 51.7 \\
14 & 56.4 & 58.2 & 53.6 & 56.1 & 53.4 \\
15 & 23.0 & 68.6 & 22.6 & 21.8 & 22.6 \\
16 & 40.5 & 52.3 & 40.4 & 40.5 & 40.5 \\
17 & 81.4 & 81.3 & 81.3 & 81.4 & 81.3 \\
18 & 20.9 & 22.9 & 20.9 & 20.8 & 20.9 \\
19 & 9.6 & 9.7 & 9.5 & 9.7 & 9.54 \\
20 & 108.8 & 109.1 & 109.2 & 109.3 & 109.3 \\
21 & 89.1 & 88.7 & 89.0 & 89.1 & 89.0 \\
22 & 74.8 & 74.0 & 74.8 & 74.8 & 74.9 \\
\hline & & & & &
\end{tabular}

$m / z(\%): 342.1\left[\mathrm{M}^{+}\right]$(2.2), $324.2(4.8), 306.1$ (6.9), 295.2 (2.7), 256.1 (7.0), 227.1 (100.0); ${ }^{1} \mathrm{H}$ NMR ( $\mathrm{CD}_{3} \mathrm{OD}, 600 \mathrm{MHz}$ ) Table $1 ;{ }^{13} \mathrm{C}$ NMR $\left(\mathrm{CD}_{3} \mathrm{OD}, 125 \mathrm{MHz}\right)$; Table 2 .

2.4.2. 13-Ethyl-11-methylene-18,19-dinor-17 $\alpha$-pregn-4-en-20-yn$6 \beta-15 \beta-17 \beta$-triol (3)

White solid; m. p. $241-243^{\circ} \mathrm{C} ;[\alpha]_{\mathrm{D}}^{25}=-299$ ( c 0.001, MeOH); IR $\left(\mathrm{CHCl}_{3}\right): v_{\max }\left(\mathrm{cm}^{-1}\right)$ 3388, 3294, $(\mathrm{OH}), 2935(\mathrm{CH}), 1641(\mathrm{C}=\mathrm{C})$; HREI-MS $\mathrm{m} / \mathrm{z} 342.2185\left[\mathrm{M}^{+}\right]\left(\mathrm{C}_{22} \mathrm{H}_{30} \mathrm{O}_{3}\right.$, calcd. 342.2195); EI-MS $m / z$ (\%): $342.3\left[\mathrm{M}^{+}\right]$(41), 324.2 (61), 277.2 (78), 241.2 (69), 133.0 (100); ${ }^{1} \mathrm{H}$ NMR $\left(\mathrm{CD}_{3} \mathrm{OD}, 600 \mathrm{MHz}\right)$ Table $1 ;{ }^{13} \mathrm{C}$ NMR $\left(\mathrm{CD}_{3} \mathrm{OD}\right.$, $125 \mathrm{MHz}$ ) Table 2.

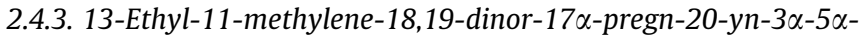
$6 \beta$-17 $\beta$-tetraol (4)

White solid; m. p. $239-242{ }^{\circ} \mathrm{C}$; $[\alpha]_{D}^{25}=-282($ c 0.001, MeOH); IR $\left(\mathrm{CHCl}_{3}\right): v_{\max }\left(\mathrm{cm}^{-1}\right) 3379,3255(\mathrm{OH}), 2929(\mathrm{CH}), 1637(\mathrm{C}=\mathrm{C})$; HREI-MS m/z $360.2305\left[\mathrm{M}^{+}\right]\left(\mathrm{C}_{22} \mathrm{H}_{30} \mathrm{O}_{4}\right.$, calcd. 360.2301); EI-MS $m / z$ (\%): $360.2\left[\mathrm{M}^{+}\right]$(09), 342.3 (17), 277.2 (37), 133.1 (48), 91.1 (65); ${ }^{1} \mathrm{H}$ NMR ( $\left.\mathrm{CD}_{3} \mathrm{OD}, 500 \mathrm{MHz}\right)$ Table $1 ;{ }^{13} \mathrm{C}$ NMR $\left(\mathrm{CD}_{3} \mathrm{OD}, 125\right.$ $\mathrm{MHz}$ ) Table 2.

2.4.4. 13-Ethyl-11-methylene-18,19-dinor-17 $\alpha$-pregn-4-en-20-yn$6 \beta$-17 $\beta$-dihydroxy-3-one (5)

White solid; m. p. $234-236{ }^{\circ} \mathrm{C}$; IR $\left(\mathrm{CHCl}_{3}\right): v_{\max }\left(\mathrm{cm}^{-1}\right) 3379$, $3255(\mathrm{OH}), 2929(\mathrm{CH}), 1637(\mathrm{C}=\mathrm{C})$; EI-MS m/z (\%): $340.3\left[\mathrm{M}^{+}\right]$ (56), 322.3 (62), 254.2 (77), 133.0 (100), 90.9 (70); ${ }^{1} \mathrm{H}$ NMR $\left(\mathrm{CD}_{3} \mathrm{OD}, 500 \mathrm{MHz}\right)$ Table $1 ;{ }^{13} \mathrm{C}$ NMR $\left(\mathrm{CD}_{3} \mathrm{OD}, 125 \mathrm{MHz}\right)$ Table 2.

\subsection{Anti-bacterial activity evaluation}

Compound 1, and its metabolites 2-5, were screened against $S$. aureus EMRSA-17, S. aureus NCTC 13277, S. aureus NCTC 13143, clinically isolated $S$. aureus, and vancomycin-resistant $S$. aureus ATCC 700699 by using in vitro assay, MABA (Microplate Alamar Blue Assay). Whereas vancomycin was used as standard drug.

In this assay, bacterial cells were grown in Muller Hinton medium and inoculums were adjusted to 0.5 McFarland turbidity index. Stock solutions of all test compounds 1-5 were prepared in DMSO and dispensed in 96-well plates up to the volume of $200 \mu$ L. $5 \times 10^{6}$ bacterial cells were added in all wells, negative control, and test compounds. The plates were sealed with parafilm and incubated for 18-20 h. After incubation, alamar blue dye was dispensed in each well, followed by shaken at 80 RPM in a shaking incubator for $2-3 \mathrm{~h}$. The change in a color of alamar blue dye from blue to pink, indicated the growth of bacterial strains. The absorbances were recorded at 570, and $600 \mathrm{~nm}$ by using ELISA reader. The percent growth inhibition results of test compounds are presented in Table 3.

$\%$ Inhibition $=\frac{(O X) \lambda 2 A \lambda 1-(O X) \lambda 1 A \lambda 2}{\left(\text { RED) } \lambda 1 A \lambda 2-(\text { RED }) \lambda 2 A^{\prime} \lambda 1\right.} \times 100$

Whereas;

$\mathrm{OX}=$ Molar Extinction coefficient of Alamar Blue oxidized form (Blue)

RED = Molar Extinction coefficient of Alamar Blue reduced form (Pink)

$\mathrm{A}=$ Absorbance of test wells

$\mathrm{A}^{\prime}=$ Absorbance of negative control well

$\lambda 1=570 \mathrm{~nm}$

$\lambda 2=600 \mathrm{~nm}$

\section{Results and discussion}

Fermentation of desogestrel $(\mathbf{1}),\left(\mathrm{C}_{22} \mathrm{H}_{30} \mathrm{O}, \mathrm{m} / \mathrm{z} 310.2\right)$, with $C$. blakesleeana yielded three new metabolites $\mathbf{2 - 4}$, along with a known compound 5.

The HREI-MS of compound 2 showed the $\left[\mathrm{M}^{+}\right]$at $\mathrm{m} / z 342.2179$ $\left(\mathrm{C}_{22} \mathrm{H}_{30} \mathrm{O}_{3}\right.$, calcd. 342.2195), indicating addition of two oxygen atoms as hydroxyl groups in substrate $\mathbf{1}(\mathrm{m} / \mathrm{z} 310)$. The IR absorbances at $3872,3393,3303$, and $1644 \mathrm{~cm}^{-1}$ were due to the presence of $\mathrm{OH}$, and $\mathrm{C}=\mathrm{C}$ groups, respectively. The ${ }^{13} \mathrm{C}-\mathrm{NMR}$ spectrum of compound 2 showed additional signals for two new methine carbons at $\delta 74.2$ and 68.6 as compared to substrate 1, indicating the hydroxylation of substrate 1 (Table 2). Similarly, two new signals for methine protons at $\delta 4.18$, and 4.16 further supported the presence of hydroxyl containing methine groups in substrate $\mathbf{1}$. The signals for methylene protons of C-6 and C-15 were also found missing in the ${ }^{1} \mathrm{H}$-NMR spectrum (Table 1 ) of metabolite 2 . The first $\mathrm{OH}$ group was placed at C-6 $(\delta 74.2)$, based on HMBC correlations of $\mathrm{H}-8$ ( $\delta 2.14$, overlap), $\mathrm{H}-4\left(\delta 5.71\right.$, br. s) and $\mathrm{H}_{2}-7(\delta 2.13$, overlap; 1.21, overlap) with C-6 ( $\delta 74.2)$ (Fig. 2). This was further supported by COSY-dfqf correlations of $\mathrm{H}_{2}-7$ ( $\delta$ 2.13, overlap;

Table 3

Anti-bacterial activity of compounds 1-5 against Staphylococcus aureus.

\begin{tabular}{|c|c|c|c|c|c|}
\hline Compounds [20 $\mu \mathrm{g} / \mathrm{mL}]$ & $\begin{array}{l}\text { S. aureus EMRSA-17 } \\
\text { (\% inhibition) }\end{array}$ & $\begin{array}{l}\text { S. aureus NCTC } 13277 \\
\text { (\% inhibition) }\end{array}$ & $\begin{array}{l}\text { S. aureus NCTC } 13143 \\
\text { (\% inhibition) }\end{array}$ & $\begin{array}{l}\text { S. aureus clinical } \\
\text { (\% inhibition) }\end{array}$ & $\begin{array}{l}\text { S. aureus (VRSA) ATCC } 700699 \\
\text { (\% inhibition) }\end{array}$ \\
\hline 1 & 92 & 82 & 88 & 89 & 74 \\
\hline 2 & 84 & 86 & 94 & 87 & - \\
\hline 3 & 27 & - & 26 & - & - \\
\hline 4 & - & - & - & - & - \\
\hline 5 & 25 & - & 30 & - & 24 \\
\hline Standard drug, vancomycin & 90 & 88 & 92 & 91 & 65 \\
\hline
\end{tabular}




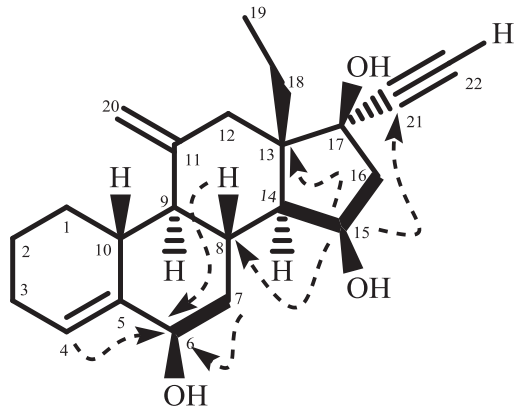

2

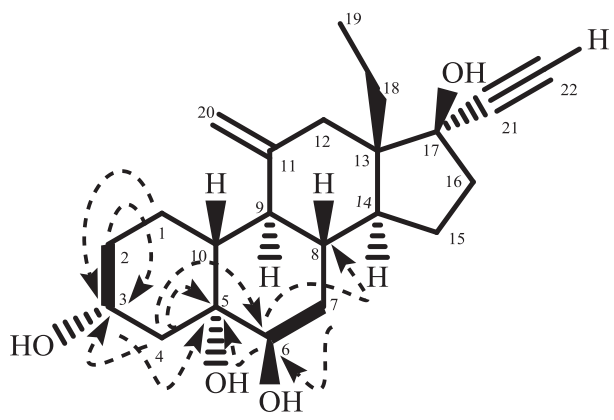

4

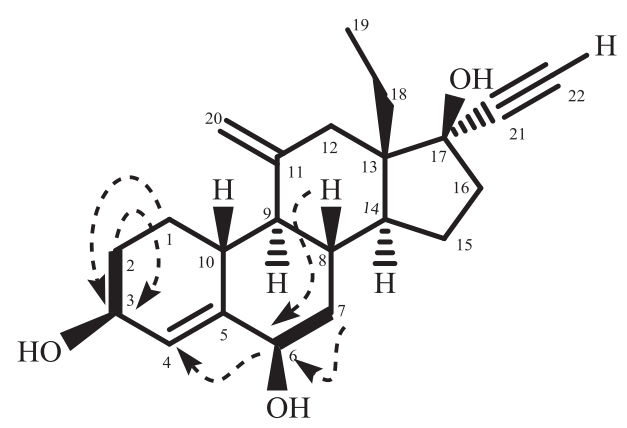

3

Fig. 2. Key HMBC (-- - $>$ ), and COSY (

1.21, overlap) with H-6 ( $\delta 4.18$, overlap) (Fig. 2$)$. The OH group at C$6(\delta$ 74.2) was deduced to be $\beta$-oriented (axial), based on NOESY correlations of equatorially-oriented H-6 ( $\delta$ 4.18, overlap) with vinylic $\mathrm{H}-4$ ( $\delta 5.71$, br. s) and $\mathrm{H}_{2}-7$ ( $\delta 2.13$, overlap; 1.21 , overlap). This can only be possible when H-6 is equatorial (Fig. 3). The half-width of equatorially-oriented H-6 $\left(W_{1 / 2}=6.4 \mathrm{~Hz}\right)$ also a $\beta$-orientation (axial) of geminal $\mathrm{OH}$ group at $\mathrm{C}-6$. The second $\mathrm{OH}$ group was placed at C-15 ( $\delta$ 68.6), based on HMBC correlations of $\mathrm{H}-15$ ( $\delta 4.16$, overlap) with C-8 ( $\delta$ 33.6), C-13 ( $\delta$ 51.5) and C-21 ( $\delta 88.7)$ (Fig. 2). This was further supported by COSY-dfqf correlations of $\mathrm{H}-15$ ( $\delta 4.16$, overlap) with $\mathrm{H}_{2}-16$ ( $\delta 2.71$, overlap; 2.12 , overlap) and $\mathrm{H}-14$ ( $\delta 1.66$, overlap) (Fig. 2$)$. The $\mathrm{OH}$ group at $\mathrm{C}-15(\delta$ 68.6) was deduced to be $\beta$-oriented, based on NOESY correlations of H-15 ( $\delta 4.16$, overlap) with axially-oriented H-14 ( $\delta 1.66$, overlap) (Fig. 3). Thus the structure of new metabolite 2 was deduced as 13-ethyl-11-methylene-18,19-dinor-17 $\alpha$-pregn-4-en-20-yn$6 \beta, 15 \beta, 17 \beta$-triol.

The HREI-MS of metabolite 3 showed the $\left[\mathrm{M}^{+}\right]$at $\mathrm{m} / z 342.2185$ (calcd. 342.2195, $\mathrm{C}_{22} \mathrm{H}_{30} \mathrm{O}_{3}$ ), suggesting addition of two hydroxyl groups in compound $\mathbf{1}(\mathrm{m} / \mathrm{z} 310)$. The IR absorbances at 3388 , 3294 , and $1641 \mathrm{~cm}^{-1}$ were due to the presence of $\mathrm{OH}$, and $\mathrm{C}=\mathrm{C}$ groups, respectively. The ${ }^{13} \mathrm{C}-\mathrm{NMR}$ spectrum showed signals for two new methine carbons at $\delta 67.8$ and 73.3, indicating addition of two hydroxyl groups in substrate $\mathbf{1}$ (Table 2). Similarly, in the ${ }^{1} \mathrm{H}-\mathrm{NMR}$ spectrum the appearance of methine proton signals at $\delta$ 4.10 , and 4.15 also indicated dihydroxylation of substrate 1 (Table 1$)$. The first $\mathrm{OH}$ group was placed at $\mathrm{C}-3(\delta 67.8)$, based on HMBC correlations of $\mathrm{H}_{2}-1$ ( $\delta 2.39$, overlap; 1.05, overlap) and $\mathrm{H}_{2}-2$ ( $\delta 1.91$, overlap; 1.38 , overlap) with C-3 $(\delta 67.8)$. This was further supported by key COSY-dfqf correlations of $\mathrm{H}-3$ ( $\delta 4.10$, overlap) with $\mathrm{H}_{2}-2$ ( $\delta 1.91$, overlap; 1.38 , overlap) (Fig. 2). The $\mathrm{OH}$ group at C-3 ( $\delta$ 67.8) was deduced to be $\beta$-oriented (equatorial), based on NOESY correlations of axially-oriented H-3 ( $\delta 4.10$, overlap) with $\mathrm{H}-2$ ( $\delta 1.91$, overlap) and $\mathrm{H}-1$ ( $\delta 1.05$, overlap). This was further supported by NOESY correlations of $\mathrm{H}-2$ ( $\delta 1.38$, overlap) with $\mathrm{H}-10(\delta 1.85, \mathrm{~m})$ (Fig. 3$)$. The half-width of axially-oriented $\mathrm{H}-3\left(W_{1 / 2}=18.9 \mathrm{~Hz}\right)$ also indicated $\beta$-orientation (equatorial) of geminal $\mathrm{OH}$ group at $\mathrm{C}-3$. The second $\mathrm{OH}$ group was placed at $\mathrm{C}$ $6(\delta 73.3)$, based on HMBC correlations of H-6 ( $\delta 4.15$, br. s) with C-4 ( $\delta$ 129.7), and H-8 ( $\delta$ 2.67, overlap) with C-6 ( $\delta$ 73.3). This was further supported by COSY-dfqf correlations of H-6 $(\delta 4.15$, br. s) with $\mathrm{H}_{2}-7$ ( $\delta 1.89$, overlap; 1.12 , overlap) (Fig. 2). The $\mathrm{OH}$ group at C-6 ( $\delta$ 73.3) was deduced to be $\beta$-oriented (axial), based on NOESY correlations of equatorially-oriented geminal H-6 ( $\delta$ 4.15 , br. s) with $\mathrm{H}_{2}-7$ ( $\delta 1.89$, overlap; 1.12 , overlap) and vinylic $\mathrm{H}-4$ ( $\delta 5.51$, br. s) (Fig. 3). This can only be possible when $\mathrm{H}-6$ is equatorial (Fig. 3). The half-width of equatorially-oriented broad singlet of H-6 $\left(W_{1 / 2}=6.3 \mathrm{~Hz}\right)$ also indicated $\beta$-orientation (axial) of geminal $\mathrm{OH}$ group at $\mathrm{C}-6$. Thus the structure of new metabolite 3 was deduced as 13-ethyl-11-methylene-18,19-dinor-17 $\alpha$-pregn4-en-20-yn-3 $\beta, 6 \beta, 17 \beta$-triol.

The HREI-MS of compound 4 showed the $\left[\mathrm{M}^{+}\right]$at $m / z 360.2305$ $\left(\mathrm{C}_{24} \mathrm{H}_{30} \mathrm{O}_{4}\right.$, calcd. 360.2301), indicating an addition of three hydroxyl groups in substrate $\mathbf{1}(\mathrm{m} / \mathrm{z} 310)$. The overlapped IR absorbances at 3379 , and $3255 \mathrm{~cm}^{-1}$ were due to the presence of hydroxyl groups. The ${ }^{13} \mathrm{C}-\mathrm{NMR}$ spectrum showed a signal for new quaternary carbon at $\delta 76.1$, along with two new methine signals at $\delta$ 69.6, and 74.4, suggesting the presence of hydroxyl groups in substrate 1 (Table 2). Similarly, new signals for methine protons at $\delta$ 3.91, and 3.41 further supported the hydroxylation of substrate 1. The signals for methylene protons of $\mathrm{C}-3$ and $\mathrm{C}-6$ were also found missing in the ${ }^{1} \mathrm{H}$ NMR spectrum. The signals for vinylic protons were also found missing in the ${ }^{1} \mathrm{H}-\mathrm{NMR}$ spectrum of metabolite 4 (Table 1 ). The first OH group was placed at C-3 ( $\delta 69.6)$, based 


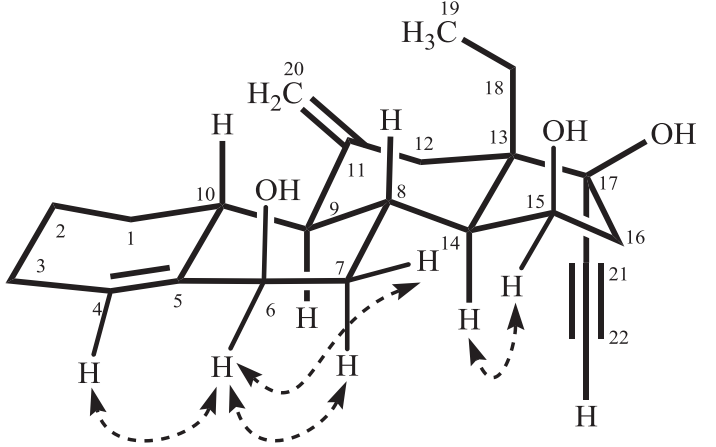

2

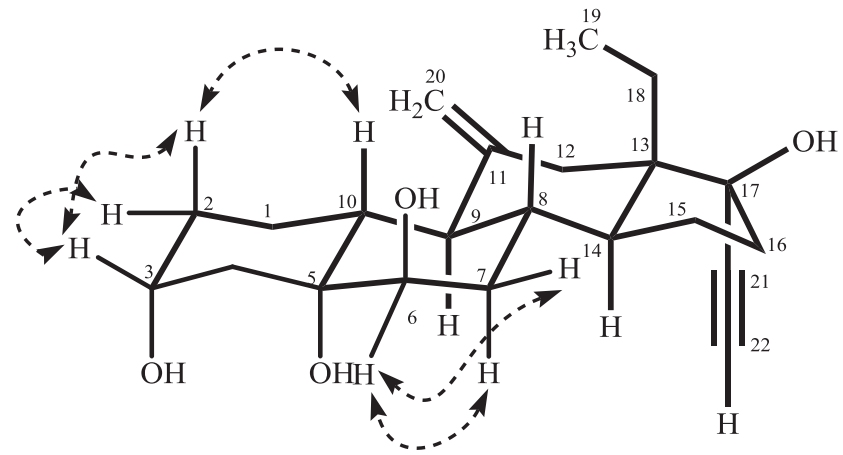

4

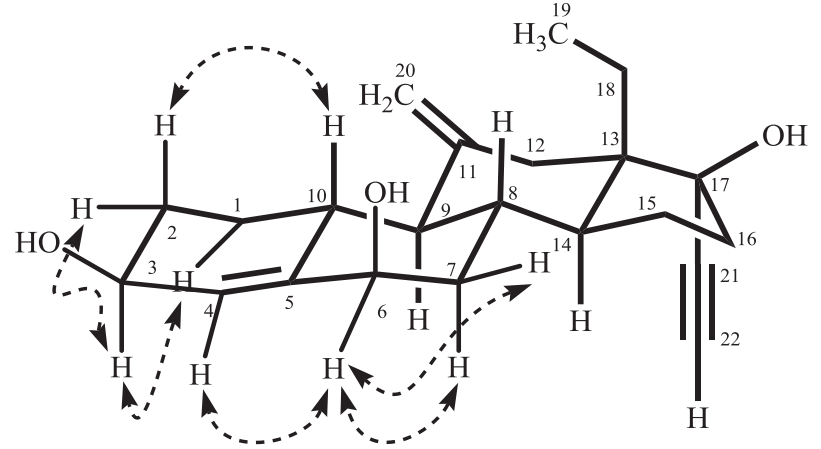

3

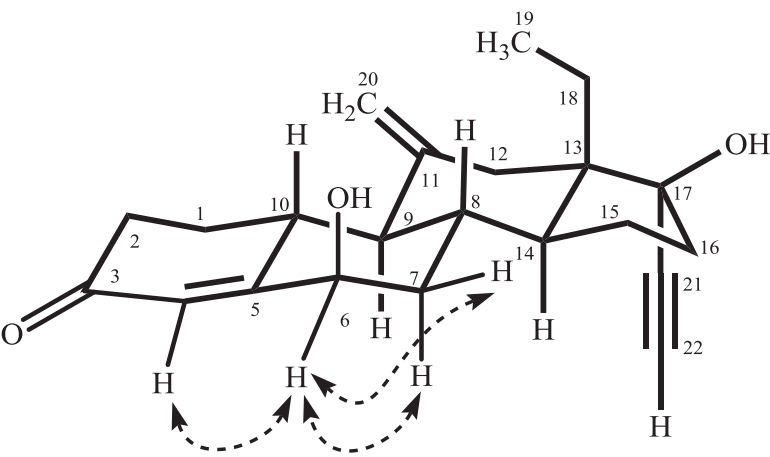

5

Fig. 3. Key NOESY correlations in metabolites $2-5$.

on key HMBC correlations of $\mathrm{H}_{2}-1$ ( $\delta 1.23$, overlap) and $\mathrm{H}-4$ ( $\delta 1.64$, overlap) with C-3 ( $\delta$ 69.6). This was further inferred from key COSY-dfqf correlations of $\mathrm{H}-3\left(\delta 3.91\right.$, br. d, $J_{e / e}=2.5 \mathrm{~Hz}$ ) with $\mathrm{H}_{2^{-}}$ 2 ( $\delta 1.91$, overlap; 1.27 , overlap) and $\mathrm{H}_{2}-4$ ( $\delta 1.90$, overlap; 1.64 , overlap) (Fig. 2). The $\mathrm{OH}$ group at $\mathrm{C}-3(\delta 69.6)$ was deduced to be $\alpha$-oriented (axial), based on NOESY correlations of equatoriallyoriented H-3 ( $\delta 3.91$, br. d, $J_{e / e}=2.5 \mathrm{~Hz}$ ) with $\mathrm{H}_{2}-2(\delta 1.91$, overlap; 1.27 , overlap) and $\mathrm{H}_{2}-4$ ( $\delta 1.90$, overlap; 1.64 , overlap). This was further supported by NOESY correlations of $\mathrm{H}-2$ ( $\delta 1.27$, overlap) with $\mathrm{H}-10$ ( $\delta 1.88$, overlap). The half-width of equatoriallyoriented broad doublet of $\mathrm{H}-3\left(W_{1 / 2}=9.6 \mathrm{~Hz}\right)$ also indicated $\alpha$ orientation (axial) of geminal $\mathrm{OH}$ group at C-3 (Fig. 3). The second $\mathrm{OH}$ group was placed at C-5 $(\delta 76.1)$, based on key HMBC correlations of $\mathrm{H}-3$ ( $\delta 3.91$, br. d), $\mathrm{H}_{2}-4$ ( $\delta 1.90$, overlap; 1.64 , overlap) and H-6 ( $\delta 3.41$, br. $\mathrm{t}$ ) with $\mathrm{C}-5(\delta 76.1)$. The $\mathrm{OH}$ group at $\mathrm{C}-5$ was deduced to be $\alpha$-oriented (axial), based on NOESY correlations of axially-oriented $\mathrm{OH}-3(\delta 4.62, \mathrm{~m})$ with axially-oriented $\mathrm{OH}-5(\delta$ 2.81 , overlap) (acetone- $d_{6}$ ) (Fig. 3 ). The third $\mathrm{OH}$ group was placed at C-6 $(\delta 74.4)$, based on key HMBC correlations of $\mathrm{H}-6(\delta 3.41$, br. $\mathrm{t})$ with C-5 ( $\delta 76.1)$, and C-8 ( $\delta 38.7)$. This was further supported by key COSY-dfqf correlations of H-6 $\left(\delta 3.41\right.$, br. t, $\left.J_{6 e / 7 e}=2.5 \mathrm{~Hz}\right)$ with $\mathrm{H}_{2}-7$ ( $\delta 1.92$, overlap; 1.52 , overlap) (Fig. 2$)$. The OH group at C-6 ( $\delta$ 74.4 ) was deduced to be $\beta$-oriented (axial) based on NOESY correlations of $\mathrm{H}-6\left(\delta 3.41\right.$, br. $\mathrm{t}, J_{6 e / 7 e}=2.5 \mathrm{~Hz}$ ) with $\mathrm{H}_{2}-7$ ( $\delta 1.92$, overlap; 1.52, overlap) (Fig. 3). The half-width of equatorially-oriented broad triplet of $\mathrm{H}-6\left(W_{1 / 2}=6.7 \mathrm{~Hz}\right)$ also indicated $\beta$-orientation (axial) of geminal $\mathrm{OH}$ group at $\mathrm{C}-6$. Thus the structure of the new metabolite 4 was deduced as 13-ethyl-11-methylene-18,19-dino r-17 $\alpha$-pregn-20-yn-3 $\alpha, 5 \alpha, 6 \beta, 17 \beta$-tetraol.

Compound 5 was identified as 13-ethyl-11-methylene-18,19-d inor-17 $\alpha$-pregn-4-en-20-yn-6 $\beta$-17 $\beta$-dihydroxy-3-one which was previously reported by Verhoeven et al. through metabolism in animals body [24].

Placement of $\mathrm{OH}$ groups in desogestrel (1) is affecting its antibacterial activity. Apparently, the presence of $\mathrm{OH}$ groups at C-6 and C-15 in compound $\mathbf{2}$ increases its anti-bacterial activity only against S. aureus NCTC 13143 in comparison to substrate $\mathbf{1}$ and standard drug, vancomycin. Presence of $\mathrm{OH}$ groups at $\mathrm{C}-3$ and C-6 in compound $\mathbf{3}$ diminished its bactericidal activity against clinically isolated S. aureus, and S. aureus NCTC 13277. Presence of $\mathrm{OH}$ groups at C-3, C-5, and C-6 in compound 4, completely diminished its anti-bacterial activity. Presence of carbonyl ketone at $\mathrm{C}-3$, and $\mathrm{OH}$ group at $\mathrm{C}-6$ in compound $\mathbf{5}$ also diminished its anti-bacterial activity against clinically isolated $S$. aureus, and $S$. aureus NCTC 13277.

\section{Conclusion}

In conclusion, microbial transformations of desogestrel (1) with C. blakesleeana afforded four new metabolites 2-4, along with a known metabolite 5. Compounds 1-2 showed a potent growth inhibition against drug resistant strains of $S$. aureus. Compounds 1-5 were found to be non-cytotoxic against 3T3 normal cell line. The presented study indicated that compounds 1-2 can be further studied for their therapeutic potential against infections, caused by MDR S. aureus.

\section{Acknowledgement}

EB acknowledges University Research Board of the American University of Beirut (Lebanon) for financial support. 


\section{Appendix A. Supplementary material}

Supplementary data associated with this article can be found, in the online version, at https://doi.org/10.1016/j.bioorg.2017.12.027.

\section{References}

[1] J.D. Steckbeck, B. Deslouches, R.C. Montelaro, Expert Opin. Biol. Ther. 14 (2014)

[2] H. Xin, S. Ji, J. Peng, P. Han, X. An, S. Wang, B. Cao, Int. J. Antimicrob. Agents 49 (2017) 427.

[3] D.J. Diekema, M.A. Pfaller, F.J. Schmitz, J. Smayevsky, J. Bell, R.N. Jones, Clin. nfect. Dis. 32 (2001) S114.

[4] H. Wisplinghoff, T. Bischoff, S.M. Tallent, H. Seifert, R.P. Wenzel, M.B. Edmond, Clin. Infect. Dis. 39 (2004) 309.

[5] S.N. Banerjee, T.G. Emori, D.H. Culver, R.P. Gaynes, W.R. Jarvis, T. Horan, Am. J. Med. 91 (1991) S86.

[6] M.B. Edmond, S.E. Wallace, D.K. McClish, M.A. Pfaller, R.N. Jones, R.P. Wenzel, Clin. Infect. Dis. 29 (1999) 239.

[7] E.U. Mughal, M. Ayaz, Z. Hussain, A. Hasan, A. Sadiq, M. Riaz, M.I. Choudhary, Bioorg. Med. Chem. 14 (2006) 4704

[8] C.J. Chen, Y.C. Huang, Clin. Microbiol. Infect. 20 (2014) 605.

[9] K.B. Borges, W.D.S. Borges, R. Duran-Patron, M.T. Pupo, P.S. Bonato, I.G. Collado, Tetrahedron Asymmetry 20 (2009) 385.
[10] W.J. Jones, C.S. Mazur, J.F. Kenneke, A.W. Garrison, Environ. Sci. Technol. 41 (2007) 8301.

[11] C. Smith, Atia-tul-Wahab, M.S.A. Khan, M.S. Ahmad, D. Farran, M.I. Choudhary, E. Baydoun, Steroids 102 (2015) 39.

12] H.L. Holland, H.K. Weber, Curr. Opin. Biotechnol. 11 (2000) 547.

[13] M.I. Choudhary, S. Erum, M. Atif, R. Malik, N.T. Khan, Steroids 76 (2011) 1288

[14] K. Fotherby, Contraception 51 (1995) 3.

[15] J. Barkfeldt, A. Virkkunen, T. Dieben, Contraception 64 (2001) 295.

[16] G. Benagiano, F. Primiero, Ann. N. Y. Acad. Sci. 997 (2003) 163.

[17] M. Siddiqui, M.S. Ahmad, Atia-tul-Wahab, S. Yousuf, N. Fatima, N.N. Shaikh, Atta-ur-Rahman, M.I. Choudhary, PloS One 12 (2017) e0171476.

[18] S. Bano, Atia-tul-Wahab, S. Yousuf, A. Jabeen, M.A. Mesaik, Atta-ur-Rahman, M.I. Choudhary, PloS One 11 (2016) e0153951.

[19] M.I. Choudhary, M.Y. Mohammad, S.G. Musharraf, M. Parvez, A. Al-Aboudi, Atta-ur-Rahman, Steroids 74 (2009) 1040.

[20] E. Baydoun, M. Karam, Atia-tul-Wahab, M.S.A. Khan, M.S. Ahmad, C. Smith, R. Abdel-Masih, M.I. Choudhary, Steroids 88 (2014) 95.

[21] S.A.A. Shah, S. Sultan, N.B. Hassan, F.K.B. Muhammed, M.A.B.M. Faridz, F.B.M Hussain, M. Hussain, H.S. Adnan, Steroids 78 (2013) 1312.

[22] Atta-ur-Rahman, M.I. Choudhary, S.A.A. Shah, S.N. Khan, US Pat No. 8198041 (2012).

[23] S.H. Hu, X.F. Tian, Y.H. Sun, G.D. Han, Steroids 61 (1996) 407.

[24] C.H.J. Verhoeven, S.F.M. Krebbers, G.N. Wagenaars, R.M.E. Vos, Drug Metab. Dispos. 26 (1998) 927. 